\title{
The interaction of obesity and inflammation in breast cancer.
}

\author{
Chia-Chien Hsieh \\ Department of Human Development and Family Studies (Nutritional Science \& Education), National Taiwan Normal \\ University, Taipei, Taiwan.
}

\begin{abstract}
Obesity and overweight have closely considered with several chronic diseases. Evidence of this field has demonstrated that significantly impact on the multiple cancers progression, such as breast cancer, prostate cancer, hepatocellular carcinoma, pancreatic cancer and cervical cancer. Epidemiological studies have shown that there is about $20 \%$ of cancer related to obesity, which were affected by lifestyle including dietary behavior and physical activity. Based on these findings, obesity is considered as one of most harmful factors for the breast cancer in women, especially after menopause. Many hypotheses have been proposed to explain how obesity contributes to breast cancer development. The inflammatory cytokines produced from adipocytes is one of main reason triggers tumor microenvironment and tumor progression. Better understanding the association between adipocytes and inflammation implanting breast tumor development could apply to develop new alternative methods for prevention/cure. In this review, we summarize the concept and evidence associated to interaction of obesity-driven changes and inflammation in breast cancer microenvironment.
\end{abstract}

Keywords: Inflammation, Breast cancer, Cytokines, Obesity, Microenvironment.

Accepted August 08, 2016

\section{Introduction}

Obesity is a critical epidemiological issue, with excess adipocytes accumulation, causing physiological and even pathological complications impacted to the health [1]. The prevalence of obesity has rapidly increased in the past decades worldwide [2]. In 2014, global statistics has been done by World Health Organization, more than 1.9 billion adults are overweight and over 600 million people are obesity [3]. In US, there are more than $35 \%$ of adult are obesity [4], suggesting that obesity is a critical problem to be prevented and cured in the future. Obesity has demonstrated that are associated with other metabolic diseases and several kinds of cancer. In addition, obesity fertilizes inflammatory microenvironment, which is favorable to tumorigenesis. The interaction between obesity and inflammation in breast cancer was summarized in this article.

\section{Obesity Accompanies Low-Grade Inflammation}

Adipocytes, as fat-storage depots, locate in adipose tissue that is an active endocrine system and associated with immunological functions as well as secretion of several kinds of adipokine [5,6]. Adipose tissue is an important energy and endocrine organ while its dysfunction strongly triggers the initiate course of several diseases [7]. The most frequent prevalence of disorders in obesity-related complication such as type II DM, insulin resistance, atherosclerosis and several cancers [8]. It is well established that a systemic metabolic disorder like obesity creates a supportive environment for tumor cell proliferation. This environment continually produced insulin, endogenous hormones and various adipokines. In addition, obesity induced hypoxia, immune modulation and inflammatory cytokines accompanied to fertility angiogenesis of cancer [9].

In thin subject, some immune cells infiltrated into adipose tissue secreting the anti-inflammatory cytokines such as interleukin-4 (IL-4), IL-10 and IL-13 to support a normal metabolic function [10]. On the contrary, obesity has more immune cells infiltration, excessive fat accumulation, causes low-grade and chronic inflammatory environment. These immune cells such as macrophages trend to secret proinflammatory cytokines such as monocyte chemotactic protein-1 (MCP-1), IL-6, and tumor necrosis factor-a (TNF- $\alpha$ ). Moreover, other tumor-infiltrating lymphocytes present in breast tissue resulting in escape from immune control, which is known as cancer immunoediting [11]. The possible processes of immunoediting in malignant cells is including elimination of immunosurveillance, equilibrium of surrounding cells, and transformed cells escape immune system [11], suggesting the malignant cells easily develop to breast cancer. 
Approving evidence has shown that the obesity-related inflammation is associated with the cancer development through multiple mechanisms [12]. The local hypoxia around adipocytes in the microenvironment was proposed that contributes to this kind of low-grade inflammation. During adiposity, the group of adipocytes enlarges and accompanies surrounding vasculature forming, made the cells without sufficient oxygen, resulting in the hypoxia [13]. Adipose tissue triggers this local hypoxia is considered as one of important factors stimulating inflammatory response [14]. Subsequently, the hypoxia-inducible factor-1 $\alpha$ (HIF-1 $\alpha$ ) is activated, involving in regulating both the innate and adaptive immunity [15]. HIF-1 1 p promotes pro-inflammatory cytokines production such as TNF- $\alpha$, MCP- 1 and IL- 6 mediated M1 macrophage infiltration in the adipose tissue. Nowadays, the obesity-associated low-grade inflammation is widely accepted as an important concept in cancer pathogenesis. Nevertheless, other factors such as adipokines and hormones in obese state that relate to breast tumor microenvironment were discussed in the following section.

\section{Breast Cancer and Its Microenvironment}

The intricate interaction between tumorigenesis and the immune regulation is a critical issue attracted scientist's interest recently. On the basis of researches, the possible mechanisms have been proposed. Tumor-associated macrophages (TAM) activate tumor proliferation, matrix remodeling, metastasis, and angiogenesis as well as blunting of adaptive immunity [16]. Innate and adaptive immunity play an important role with the dynamic interaction resulting in tumor progression or inhibition [17]. In normal physiology, macrophages play an important role in host defense and in proper tissue development [18]. These cells, product several cytokines, directly act on the inflammatory developmental processes in the innate immune system, and these mediators also bridge to the adaptive immune system, contributing to pathogens clearance, sensors of tissue damage and maintaining tissues homeostasis [18]. In tumor microenvironment, neoplastic cells secret tumor-derived chemotactic factors like MCP-1, vascular endothelial growth factor (VEGF) and macrophage colony stimulating factor (M-CSF) recruiting circulating monocytes, and then trigger the monocytes differentiation toward TAM. Experimental animal and human studies were demonstrated these mediators linked to poor prognosis in ovarian, breast and endometrial cancers [19].

Macrophages are polarized and divided to two phenotypes, the M1 and M2 macrophage with different characteristics [20]. M1 macrophage is promoted by T helper type I (Th1) cytokines such as IL- 1 and TNF- $\alpha$ and Toll-like receptor (TLR) ligands to produce inflammatory cytokines, thereby, brings of the adaptive immune response as well as defend the viral and microbial infections in host. Regard to the M2 macrophage, is promoted by Th2 cytokines like IL-4 and IL-13, exerts angiogenesis, wound healing, injured tissues cleaning, and blunts the adaptive immune response [21]. The imbalance of population between M1 and M2 macrophages linked to pathological development. For example, uncontrolled counts polarization of M1 macrophage induces chronic inflammatory diseases while excessive M2 macrophage promotes immune suppression [22]. Furthermore, TAM seems like M2 macrophage, which executes the tumorigenesis properties in the microenvironment [23]. The murine tumor models have demonstrated that TAM executes tumor promotion via nuclear transcription factor $(\mathrm{NF}-\kappa \mathrm{B})$ signaling pathway [24]. Otherwise, the local hypoxia activates the HIF$1 \alpha$ around TAM of tumors was also showed through the activation of NF- $\kappa \mathrm{B}$. Moreover, TAM alternately produces cytokines and chemokine's sustaining and amplifying the neoplastic favor environment [25]. The variation of immune system during malignance procession highlights that diverse immune regulations in the tumor microenvironment play an important role connected with inflammation and cancer.

\section{Obesity and Breast Cancer}

On the basis of epidemiological and experimental studies, the obesity development links with metabolic diseases and several kinds of cancer. The excess adipose tissue causes neoplastic status developed to cancer such as endometrium, breast, esophagus, liver, colon and ovary [7]. Especially, some study was showed the close relationship between abnormal obesity and breast cancer [1]. Obesity is associated with a worse breast cancer prognosis and increases the expression of cyclooxygenase-2 (COX-2) and the infiltration of macrophages in the adipose tissue. Eicosanoid prostaglandin E2 (PGE2) is the pro-inflammatory mediator, which is a downstream factor of COX-2, stimulates aromatase expression and estrogen production in adipose tissue, thereby, promotes breast cancer development [26].

\section{Factors Involved in Obesity-Associated Breast Cancer}

Several kinds of cell, extracellular matrix, soluble factors and signaling molecules are components in the tumor microenvironment, which stimulate tumorigenesis and escape the immune response. In addition, accumulating obese tissue diminishes the oxygen level leading to hypoxia, resulting in modification the genes expression related to angiogenesis, cell proliferation and apoptosis, and ultimately increases the risk of cancer development in this kind of microenvironment [14]. A growing body of obesity-related inflammatory state is associated with promoting cancer development. Nevertheless, other factors such as estrogen, insulin-like growth factor-1 (IGF-1) and leptin involving in obesity-associated breast cancer were summarized below.

An increase of estrogen synthesis from adipocytes as well as raises the levels of insulin and IGF-1 secretions were accompanied with obesity [27]. In premenopausal women, estrogen is usually synthesized by ovaries, however, in obese postmenopausal women, estrogen is major produced from excessive adipose tissue and this kind of estrogen promotes the breast cancer development [28]. In addition, over expressed Insulin, IGF-1 and IGF-1 receptor (IGF-1R) are present in many kinds of cancer such as obesity-associated breast cancer [29].

Leptin and adiponectin are two major adipokines secreted by adipocytes associated with breast cancer development. Leptin, a hormone peptide, exerts satiety and energy homeostasis, has also been found that obese subjects have higher level of leptin in serum compared to the lean individuals. The possible actions of leptin are involving in cell survival, proliferation, 
angiogenesis, immune regulation and inflammatory response, which contribute to tumor progression [30]. Furthermore, leptin has been showed to crosstalk with the estrogen pathway by enhancing the expression of aromatase, thereby, increase estrogen synthesis. Some study has demonstrated that leptin induced reactive oxygen species (ROS) production and caused COX-2 expression and NF- $\kappa \mathrm{B}$ pathway activation and then increased several pro-inflammatory mediators' secretion [31]. On the contrary, adiponectin is another adipokine that inversely correlates with adiposity. Several clinical evidences were reported that adiponectin acted a protective effect on the procession of breast cancer [32]. Therefore, the contrary roles between leptin and adiponectin were indicated in cancer development as leptin affects tumor development and progression, while adiponectin involves in tumor suppression.

In summary, accumulating evidence related to obesity and breast cancer has been proposed continually. To explore advance and effective treatments for prevention and/or cure in obesity-related breast tumorigenesis could be applied to increase the life quality of patients with this kind of cancer. Therefore, a comprehensive cooperation among the relevant epidemiologists and statisticians, researchers and clinicians is critical needed to approach this goal.

\section{Acknowledgement}

Chia-Chien Hsieh acknowledges funding from Ministry of Science and Technology, Taiwan (MOST 103-2320-B-003003-MY3).

\section{References}

1. Strong AL, Ohlstein JF, Biagas BA, et al. Leptin produced by obese adipose stromal/stem cells enhances proliferation and metastasis of estrogen receptor positive breast cancers. Breast Cancer Research 2015; 17: 112.

2. Finucane MM, Stevens GA, Cowan MJ, et al. National, regional and global trends in body-mass index since 1980: Systematic analysis of health examination surveys and epidemiological studies with 960 country-years and 9.1 million participants. Lancet (London, England) 2011; 377: 557-567.

3. World Health Organization (WHO) Media Center, Geneva. Obesity and overweight. Facts about overweight and obesity 2015.

4. Flegal KM, Carroll MD, Kit BK, et al. Prevalence of obesity and trends in the distribution of body mass index among US adults, 1999-2010. JAMA 2012; 307: 491-497.

5. Lago F, DieguezC, Gomez-Reino J, et al. Adipokines as emerging mediators of immune response and inflammation. Nature Clinical Practice Rheumatolog 2007; 3: 716-724.

6. Ahima RS. Adipose tissue as an endocrine organ. Obesity (Silver Spring, Md.).2006; 242s-249s.

7. Louie SM, Roberts LS, Nomura DK. Mechanism's linking obesity and cancer. Biochimica et Biophysica Acta 2013; 1831: 1499-1508.

8. Aggarwal BB, Gehlot P. Inflammation and cancer: How friendly is the relationship for cancer patients? Current Opinion in Pharmacology 2009; 9: 351-369.
9. Fukumura D, Incio J, Shankaraiah RC, et al. Obesity and cancer: An angiogenic and inflammatory link. Microcirculation 2016; 23: 191-206.

10. Catalan V, Gomez-Ambrosi J, Rodriguez A, et al. Adipose tissue immunity and cancer. Frontiers in Physiology 2013; 4: 275 .

11. Dushyanthen S, Beavis PA, Savas P, et al. Relevance of tumor-infiltrating lymphocytes in breast cancer. BMC Medicine 2015; 24: 202.

12. Hursting SD, Dunlap SM. Obesity, metabolic dysregulation and cancer: A growing concern and an inflammatory (and micro environmental) issue. Annals of the New York Academy of Sciences 2012; 1271: 82-87.

13. Neels JG, Olefsky JM. Inflamed fat: What starts the fire? The Journal of Clinical Investigation 2006; 116: 33-35.

14. Trayhurn P. Hypoxia and adipose tissue function and dysfunction in obesity. Physiological Reviews 2013; 93: $1-21$.

15. Eltzschig HK, Carmeliet P. Hypoxia and inflammation. The New England Journal of Medicine. 2011; 364: 656-665.

16. Qian BZ, Pollard JW. Macrophage diversity enhances tumor progression and metastasis. Cell 2010; 141: 39-51.

17. Allavena P, Mantovani A. Immunology in the clinic review series; focus on cancer: Tumour-associated macrophages: Undisputed stars of the inflammatory tumour microenvironment. Clinical and Experimental Immunology 2012; 167: 195-205.

18. Brady NJ, Chuntova P, Schwertfeger KL. Macrophages: Regulators of the inflammatory microenvironment during mammary gland development and breast cancer. Mediators of Inflammation 2016:45: 13.

19. Solinas G, Germano G, Mantovani A, et al. Tumor-associated macrophages (TAM) as major players of the cancer-related inflammation. Journal of Leukocyte Biology 2009; 86: 1065 1073.

20. Gordon S, Taylor PR. Monocyte and macrophage heterogeneity. Nature reviews. Immunology 2005; 5: 953964.

21. Gordon S, Martinez FO. Alternative activation of macrophages: Mechanism and functions. Immunity 2010; 32: 593-604.

22. Condeelis J, Pollard JW. Macrophages: Obligate partners for tumor cell migration, invasion and metastasis. Cell 2006; 124: $263-266$.

23. Van Ginderachter JA, Movahedi K, Hassanzadeh GG, et al. Classical and alternative activation of mononuclear phagocytes: Picking the best of both worlds for tumor promotion. Immunobiology 2006; 211: 487-501.

24. Biswas SK, Lewis CE. NF-kappaB as a central regulator of macrophage functions in tumors. Journal of Leukocyte Biology 2010; 88: 877-884.

25. Mantovani A, Allavena P, Sica A, et al. Cancer-related inflammation. Nature 2008; 454: 436-444.

26. Bowers LW, Maximo IX, Brenner AJ, et al. NSAID use 
reduces breast cancer recurrence in overweight and obese women: role of prostaglandin-aromatase interactions. Cancer Research 2014; 74: 4446-4457.

27. Maccio A, Madeddu C, Mantovani G. Adipose tissue as target organ in the treatment of hormone-dependent breast cancer: New therapeutic perspectives. Obesity Reviews 2009; 10: 660-670.

28. Carmichael AR. Obesity as a risk factor for development and poor prognosis of breast cancer. BJOG: An International Journal of Obstetrics and Gynaecology 2006; 113: 11601166 .

29. Law JH, Habibi G, Hu K, et al. Phosphorylated insulin-like growth factor-i/insulin receptor is present in all breast cancer subtypes and is related to poor survival. Cancer Research 2008; 68: 10238-10246.

30. Delort L, Rossary A, Farges MC, et al. Leptin, adipocytes and breast cancer: Focus on inflammation and anti-tumor immunity. Life Sciences 2015; 140: 37-48.

31. Tian YF, Chang WC, Loh $\mathrm{CH}$, et al. Leptin-mediated inflammatory signaling crucially links visceral fat inflammation to obesity-associated beta-cell dysfunction. Life Sciences 2014; 116: 51-58.

32. Tworoger SS, Eliassen AH, Kelesidis T, et al. Plasma adiponectin concentrations and risk of incident breast cancer. The Journal of Clinical Endocrinology and Metabolism 2007; 92: 1510-1516.

\section{Correspondence to:}

Chia-Chien Hsieh, Ph.D.,

Department of Human Development and Family Studies, National Taiwan Normal University,

No. 162, Heping East Road,

Section 1, Taipei 10610,

Taiwan.

Tel: +886-2-7734-1439

Fax: +886-2-2363-9635

E-mail: hsieh@ntnu.edu.tw 